\title{
Role of Densitometric Criteria in Evaluation of Effectiveness of Antiangiogenic Therapies in Metastatic Colorectal Cancer: An Italian Clinical Experience
}

\author{
GIUSEPPE CICERO ${ }^{1}$, GIUSEPPE LO RE ${ }^{2}$, ROSSELLA DE LUCA ${ }^{1}$, FEDERICA VERNUCCIO ${ }^{2}$, \\ DARIO PICONE ${ }^{2}$, MASSIMO MIDIRI ${ }^{2}$ and ROBERTO LAGALLA ${ }^{2}$ \\ ${ }^{1}$ Department of Surgical, Oncological and Oral Sciences, \\ Section of Medical Oncology, University of Palermo, Palermo, Italy; \\ ${ }^{2}$ Department of Radiology, University of Palermo, Palermo, Italy
}

\begin{abstract}
Background/Aim: To evaluate the role of densitometric criterion using the Choi Criteria in the assessment of the response to antiangiogenic treatments of metastatic colorectal cancer ( $m C R C$ ) compared to the RECIST criteria. Patients and Methods: Fifty-four patients (mean age $=50.6$ years ) affected by advanced colorectal cancer and with hepatic and possibly peritoneal and pulmonary metastases, that can be treated with bevacizumab, were prospectively evaluated by computerized tomography (CT) scan. Metastases were also evaluated by $C T$ in onedimensional form according to RECIST. Results: Results show that in 58\% of analyzed cases, stable disease according to RECIST coincided with stable disease according to the CHOI criteria, whereas in $42 \%$ of analyzed cases disease progression according to RECIST corresponded to stable disease or even partial response according to CHOI criteria. Conclusion: By using the densitometric criterion with $\mathrm{CHOI}$ criteria, the evaluation of the response to antiangiogenic treatment of $m C R C$ is partially different compared to RECIST criteria.
\end{abstract}

The discovery and use of new target therapies in oncology revolutionized the treatment of solid tumors, allowing oncologists to tailor cancer therapies on patients and, above all, tumor's intrinsic characteristics, thus maximizing their therapeutic effects through a precise selection of responder patients and avoiding useless toxicities in non-responder patients $(1,2)$.

Correspondence to: Prof. Giuseppe Cicero, Department of Surgical, Oncological and Oral Sciences, University of Palermo, Via del Vespro, n. 129, 90127 Palermo, Italy. Tel: +39 0916554531, Fax: +390916552549, e-mail: giuseppe.cicero@unipa.it

Key Words: Antiangiogenic therapy, metastatic colorectal cancer (mCRC), densitometric criteria, RECIST, CHOI.
However, target therapies' mechanism of action is totally different from traditional cancer treatments because they are cytostatic rather than cytotoxic. Thus, response to these treatments is totally different (3). Since 2001 response to cancer therapies is evaluated through the Response Evaluation Criteria in Solid Tumors (RECIST), which are based on dimensional changes of tumor lesions after treatments (4). RECIST presupposes the distinction of tumor lesions into measurable and not measurable ones. According to RECIST, measurable lesions are those lesions that may be faithfully defined in at least one dimension (largest diameter in a single lesion, sum of largest diameters in multiple lesions) which has to be equal to or larger than $10 \mathrm{~mm}$ in computerized tomography (CT). After detecting measurable lesions, we have to identify target lesions. They are all measurable lesions up to a maximum of two lesions per organ and five lesions in total that should be representative of all involved organs. Tumor lesions with the longest largest diameter should be chosen and evaluated at baseline (5).

However, the target therapies mechanism of action is completely different from traditional cancer, causing the reduction of the tumor cellularity and density without change of size.

CHOI criteria were developed in 2007 in order to assess response of gastrointestinal stromal tumors (GISTs) to imatinib. CHOI criteria were also proved as better predictors of long-term prognosis than RECIST in GIST (6). However, what has been shown for first-line treatment with imatinib hasn't been confirmed for second-line treatment with sunitinib $(7,8)$. On the basis of what has been shown for the treatment of GISTs, CHOI were also studied as criteria for assessing response to therapies of HCC and kidney cancers, getting encouraging results (only when best response to the treatment of kidney cancer with sunitinib was assessed, predictive value of CHOI criteria was similar to RECIST's) $(9,10)$. Even CHOI criteria were better than RECIST in 
assessing HCC's response to trans arterial radio-embolization (11). Even in treatment of metastatic colorectal cancer (mCRC) the role of biological therapies has become more and more considerable over the years, especially in patients now defined as all RAS Wild Type, but also in those defined as all RAS mutated too. Anti-epithelial growth factor receptor (EGFR) and antiangiogenic therapies allowed researchers to get important results in terms of progressionfree-survival (PFS) and overall-survival (OS) $(12,13)$, but offered again for this condition the problem of the evaluation of response. This question occurred especially for antiangiogenic therapies. In fact, anti-EGFR target therapies are characterized by a high "tumor shrinkage" and then a high dimensional response rate, so that they are used in clinical practice as a gold standard in the so-called conversion therapies, that is when the purpose of treatment is to make resectable liver metastases which are first unresectable or when patients are highly symptomatic. Antiangiogenic therapies, instead, have a significantly lower ability to cause "tumor shrinkage", and the response to them is usually long-lasting stable disease $(14,15)$. This underlines the need to have some assessment criteria suitable to properly evaluate this kind of response.

The aim of this prospective study was to evaluate the role of densitometric criterion using the Choi Criteria in the assessment of the response to antiangiogenic treatments of mCRC compared to RECIST criteria.

\section{Patients and Methods}

Participants. All patients affected by chemo naïve advanced colorectal cancer, not susceptible to potentially curative therapies such as surgery, radiotherapy or others, distant metastases, evaluable by CT scan in one-dimensional form according to RECIST (liver metastases necessary inclusion criterion) and eligible for treatment with antiangiogenic treatment with bevacizumab because of failure of previous therapies and with good hepatic, cardiac and renal function were included. Exclusion criteria were contraindication to $\mathrm{CT}$ as kidney failure (serum creatinine $>2 \mathrm{mg} / \mathrm{dl}$ ), pregnancy and a documented allergy to iodinated contrast. Thus, 54 patients aged between 64 and 54 years (mean age 50.6 years; 27 males and 27 females) were included (Table I).

Procedure. All patients were prospectively studied with 64 slices MDCT scanner (Brilliance 64, Philips Medical System, Cleveland, Ohio, USA) by using following parameters of expansion: collimation, $64 \times 0.5 \mathrm{~mm}$, gantry's rotation time, $420 \mathrm{~ms}$, slice thickness $1.5 \mathrm{~mm}$; increase $0.7 \mathrm{~mm}, 120 \mathrm{kV}, 250 \mathrm{mAs} \mathrm{e}$ reconstruction interval $1 \mathrm{~mm}$. We used an automatic method of dose reduction in all examinations. Examinations were carried out 70 seconds after the administration of $120 \mathrm{ml}$ of non-ionic iodinated contrast (Iodixanol, Visipaque 320, GE Healthcare Srl Milano, Italia) through a peripheral vein at a speed of $3 \mathrm{ml} / \mathrm{sec}$ using a double-syringe power injector. CT scans were performed in the supine position and we obtained a scan volume from the diaphragm to pubic symphysis, during a single apnea. Patients were submitted
Table I. Baseline demographic and clinical characteristics $(n=54)$.

\begin{tabular}{lc}
\hline Number of patients & 54 \\
\hline Male & 27 \\
Female & 27 \\
K-RAS state & 36 \\
WT & 18 \\
Mutated & \\
Metastatic sites & 54 \\
Liver & 13 \\
Liver + lung & 2 \\
Peritoneal carcinomatosis & FOLFIRI + Bevacizumab (45) \\
First line treatment & FOLFOX + Bevacizumab (9) \\
&
\end{tabular}

to a CT scan at baseline before treatment and a second CT scan six months after the beginning of treatment. Lesions' size and density were determined. In the CT images, lesions' density was measured in Hounsfield Unit (HU) in the portal venous phase. Lesions' density was measured through a region of interest (ROI), delimiting the entire lesion including the hypervascularised board, if present. We alternately used an ellipsoid or circular ROI that included the entire target lesion. HU changes during the follow up were evaluated according to RECIST: HU measurements of all lesions were estimated as the average in every follow up and the resulting $\mathrm{HU}$ average value was compared to nadir of HU average values. Thereafter response to treatment was determined according to RECIST and CHOI criteria as shown in Table II.

Evaluation on the response to treatment. The assessment of the response to therapy in patients affected by metastatic colorectal cancer and treated with Bevacizumab is based on the evaluation of tumor lesions' size and density changes and possible appearance of new lesions. Tumor lesions' size should be generally measured according to RECIST. According to RECIST, complete response (RC) is defined as the disappearance of all lesions and the reduction of the smallest diameter of the lymph nodes under $10 \mathrm{~mm}$ while a partial response (RP) is defined as a reduction of at least $30 \%$ of the sum of the maximum diameters of target lesions from baseline. Stable disease (SD) is a reduction below $30 \%$ or an increase below $20 \%$ of the sum of the maximum diameters of the target lesions. The detection of new tumor lesions or the increases of at least $20 \%$ of the sum of the maximum diameters of target lesions define a progression disease (PD).

Choi criteria define complete response (RC) as the disappearance of all lesions and absence of new ones. Partial response (RP) is defined as a reduction of at least $10 \%$ of tumor lesions' size or at least $15 \%$ of tumor lesions' density, measured in HU, in the absence of new lesions and clear progression of non-measurable lesions. Increase of at least $10 \%$ of tumor lesions' size, without signs of response about their density, and/or the appearance of new lesions configure a progression disease (PD). All cadres of disease that are not covered are defined as stable disease (SD) (Table II).

The data we collected were analyzed using an advanced computer system (Elefante. NET Suite. Agfa HealthCare NV), not only on axial images, but also using multiplanar images (MPR), 
Table II. Evaluation Criteria: RECIST versus CHOI $(n=54)$.

\begin{tabular}{|c|c|c|}
\hline & RECIST criteria & CHOI criteria \\
\hline CR & Disappearance of all lesions & Disappearance of all lesions \\
\hline PR & $\begin{array}{l}\text { Tumor's size reduction } \geq 30 \% \\
\text { No new lesions }\end{array}$ & $\begin{array}{c}\text { Tumor's size reduction } \geq 10 \% \text { or tumor's density reduction } \geq 15 \% \text { in TC scan } \\
\text { No new lesions }\end{array}$ \\
\hline & No PD in target lesions & No PD in not measurable lesions \\
\hline SD & No criteria to define CR or PR or PD & No criteria to define CR or PR or PD \\
\hline & No worsening of symptoms because of tumor progression & No worsening of symptoms because of tumor progression \\
\hline PD & $\begin{array}{c}\text { Tumor's size increase } \geq 20 \% \\
\text { New lesions }\end{array}$ & $\begin{array}{c}\text { Tumor's size increase } \geq 10 \% \text { and no criteria to define PR } \\
\text { New lesions }\end{array}$ \\
\hline
\end{tabular}

$\mathrm{CR}=$ Complete response; $\mathrm{PR}=$ Partial Response; $\mathrm{SD}=$ Stable Disease; $\mathrm{PD}=$ Progression Disease according Recist and Choi criteria.

maximum intensity projections (MIP) and renderings were visualized. CT images were independently analyzed by two expert radiologists and thereafter in consensus. Between the two observers, agreement on identification and delineation of the lesions was obtained in all cases.

For each patient, three liver metastases were identified as target lesions and the total lesion was 162 .

Data were collected and analyzed in an Excel database (Microsoft, Redmond, WA, USA).

\section{Results}

Results were assessed according to both CHOI and RECIST criteria (Table II). Median follow-up time of our patients was 6 months and median duration of treatment, at the time of revaluation, was 4.5 months. We evaluated a total of 162 liver lesions. According to RECIST, no patient had a complete response, $12(22.22 \%)$ patients had a partial response, $31(57.41 \%)$ a stable disease and $11(20.37 \%)$ a disease progression. According to $\mathrm{CHOI}$ criteria, the absence of a complete response according to RECIST was confirmed, while we had $18(33.34 \%)$ partial responses, $30(55.55 \%)$ stable diseases and $6(11.11 \%)$ disease progressions. Three patients had some new lesions according to CHOI criteria, too (Table III). The median change in tumor size in the lesions of PR patients was $-44.32 \%$, in the lesions of SD patients was $-12.58 \%$ and in the lesions of PD was $26.16 \%$. The median change in tumor density in the lesions of PR patients was $-29.16 \%$, in the lesions of SD patients was $-6.9 \%$ and in the lesions of PD was $8.64 \%$ (Tables IV and $\mathrm{V})$. In support of these results, we conducted a parallel analysis about biological response through the evaluation of tumor biomarkers CEA and $\mathrm{Ca}$ 19.9. This analysis is reported below (Table VI).

By analyzing the data we collected and comparing the assessment of the response to treatment according to RECIST with the one obtained according to CHOI criteria, we observed three different scenarios:
Table III. MDTC evaluations according to RECIST and CHOI criteria.

\begin{tabular}{lcccc}
\hline Criteria & CR & PR & SD & PD \\
\hline RECIST & 0 & 12 & 31 & 11 \\
CHOI & 0 & 18 & 30 & 6 \\
\hline
\end{tabular}

$\mathrm{CR}=$ Complete response; $\mathrm{PR}=$ Partial Response; $\mathrm{SD}=$ Stable Disease; $\mathrm{PD}=$ Progression Disease according Recist and Choi criteria.

Table IV. Average values (\%) after 6 months of treatment according to RECIST and CHOI $(n=54)$.

\begin{tabular}{lcc}
\hline & Diameter (\%) & HU (\%) \\
\hline PR & $-44.32 \%$ & $-29.16 \%$ \\
SD & $-12.58 \%$ & $-6.9 \%$ \\
PD & $26.16 \%$ & $8,64 \%$ \\
\hline
\end{tabular}

$\mathrm{PR}=$ Partial Response; SD=Stable Disease; $\mathrm{PD}=$ Progression Disease according Recist and Choi criteria.

- Stable disease according to CHOI criteria and stable disease according to RECIST: in 58\% of analyzed cases we had a full agreement between RECIST and CHOI criteria about what has been detected, therefore disease was found to be stable according to both evaluation criteria and patients continued their therapy.

- Progression disease according to RECIST vs. stable disease or partial response according to CHOI criteria: this situation was observed in $40 \%$ of analyzed cases and was the most interesting one, because it showed how, by using the densitometric criterion, the evaluation of the response to antiangiogenic treatment of $\mathrm{mCRC}$ may radically change with inevitable clinical consequences. We still have to learn 
Table V. Median values before and after 6 months of treatment according RECIST and CHOI $(n=54)$.

\begin{tabular}{|c|c|c|c|c|}
\hline & \multicolumn{2}{|c|}{ Median pre-treatment values (range) } & \multicolumn{2}{|c|}{ Median values at evaluation at 6 months (range) } \\
\hline & Size (mm) & Attenuation (HUs) & Size $(\mathrm{mm})$ & Attenuation (HUs) \\
\hline PR & $24.42(12-51)$ & $69.17(43-91)$ & $13.58(8-30)$ & $49(25.8-72)$ \\
\hline SD & $60.17(21-114)$ & $68.07(43-89)$ & $52.6(18-101)$ & $63.3(40.5-80.1)$ \\
\hline PD & $62.42(21-90)$ & $63.83(43-78)$ & $78.75(28-125)$ & $69.35(51.6-91.2)$ \\
\hline
\end{tabular}

$\mathrm{PR}=$ Partial Response; $\mathrm{SD}=$ Stable Disease; $\mathrm{PD}=$ Progression Disease according Recist and Choi criteria.

how to apply the densitometric criterion and actually predict more accurately prognosis and outcome of the patients.

- Stable disease or partial response according to RECIST vs. progression disease according to CHOI criteria: this situation was observed only in $2 \%$ of analyzed cases and was not statistically significant. In fact, it is very rare that a reduction of the maximum diameter of a lesion is accompanied by an increase of its density.

\section{Discussion}

It is well established that new antineoplastic target therapies need different criteria for assessing the response from those used for traditional chemotherapy, because response to these treatments is very different. First CHOI showed this for GISTs treatment with Imatinib and second the inclusion of new criteria for assessing response that consider not only target lesions' size but also their density (16). Because antiangiogenic treatment of $\mathrm{mCRC}$ is characterized by a different response if compared to classic chemotherapy, with a higher percentage of stable disease and lower tumor shrinkage (17-19), it seems interesting to evaluate the role of the densitometric criterion in this group of patients. This analysis, in fact, shows how the response to antiangiogenic treatments of mCRC may be totally different if it is assessed according to the densitometric criteria. Many responses which according to RECIST are defined as "disease progression" should be defined as "stable disease" or even "partial response" according to densitometric criteria. Clinical consequences could be obviously very important $(20,21)$. However, CHOI criteria leave very important doubts and questions. Many tumor lesions, especially lung lesions, can be assessed only by RECIST because their diameters are lower than $15 \mathrm{~mm}$, while hypodense lesions could be difficult to assess by $\mathrm{CHOI}$ criteria regarding the attenuation (it is difficult to assess a reduction of the attenuation equal to $15 \%$ in a hypodense lesion). Reliability of CHOI criteria can also be reduced when few lesions are analyzed or tumor density is very heterogeneous and makes
Table VI. Evaluation of tumor biomarkers CEA and CA 19.9.

\begin{tabular}{lcc}
\hline Marker & $\begin{array}{c}\text { Average value } \\
\text { before treatment }\end{array}$ & $\begin{array}{c}\text { Average value } \\
\text { Follow-up (reduction \%) }\end{array}$ \\
\hline CEA & 384,8 & $124,2(-78)$ \\
CA 19.9 & 103,5 & $40.3(-61)$ \\
\hline
\end{tabular}

the assessment of attenuation very difficult $(22,23)$. We also have to underline, above all, that CHOI criteria unfortunately have a high inter-observer variability; the assessment of the response according to densitometric criteria is overly dependent on the operator. Therefore, a computerized system of evaluation of tumor attenuation that could make the assessment of tumor lesions' density as objective and reproducible as possible should be developed. Finally, this analysis shows how the assessment of the response should be changed even for antiangiogenic treatment of mCRC, in order to unequivocally identify responder patients. In $40 \%$ of analyzed cases, patients were found to be in a stable disease or partial response according to CHOI criteria, whereas RECIST defined a disease progression. However, further studies need to investigate these results in order to understand if there is a prognostic impact of this discrepancy and if CHOI criteria are actually more effective than RECIST in the evaluation of the response to antiangiogenic treatments in mCRC. Next step should be to evaluate if the difference between responders and non- responders according to CHOI criteria is really higher, for PFS and OS, than RECIST.

\section{Conclusion}

Our analysis clearly shows that response to antiangiogenic treatments leads to an important discrepancy in the evaluation of tumor response to therapy using RECIST and CHOI 
criteria. However, it is yet to be proven that CHOI criteria could replace or accompany RECIST in this group of patients.

\section{Conflicts of Interest}

The Authors have nothing to disclose.

\section{References}

1 Cicero G, De Luca R, Dorangricchia P and Dieli F: The Clinical Efficacy of Enzalutamide in Metastatic Prostate Cancer: Prospective Single-center Study. Anticancer Res 37(3): 14751480, 2017.

2 Bronte G, Silvestris N, Castiglia M, Galvano A, Passiglia F, Sortino G, Cicero G, Rolfo C, Peeters M, Bazan V, Fanale D, Giordano A and Russo A: New findings on primary and acquired resistance to anti-EGFR therapy in metastatic colorectal cancer: Do all roads lead to RAS? Oncotarget 6(28): 24780-24796, 2015.

3 Rolfo C, Fanale D, Hong DS, Tsimberidou AM, Piha-Paul SA, Pauwels P, Van Meerbeeck JP, Caruso S, Bazan V, Cicero G, Russo A and Giovannetti E: Impact of microRNAs in resistance to chemotherapy and novel targeted agents in non-small cell lung cancer. Current Pharmaceutical Biotechnology 15(5): 475485, 2014

4 Chun YS, Vauthey JN Boonsirikamchai P, Maru DM, Kopetz S, Palavecino M, Curley SA, Abdalla EK, Kaur H, Charnsangavej $\mathrm{C}$ and Loyer EM.: Association of computed tomography morphologic criteria with pathologic response and survival in patients treated with bevacizumab for colorectal liver metastases. JAMA 302(21): 2338-2344, 2009.

5 Chung WS, Park MS Shin SJ, Baek SE, Kim YE, Choi JY and Kim MJ: Response evaluation in patients with colorectal liver metastases: RECIST version 1.1 versus modified CT criteria AJR 199(4): 809-815, 2012.

6 Therasse P, Arbuck SG, Eisenhauer EA, Wanders J, Kaplan RS Rubinstein L, Verweij J, Van Glabbeke M, van Oosterom AT, Christian MC and Gwyther SG: New Guidelines to Evaluate the Response to Treatment in Solid Tumors. J Natl Canc Inst 92: 205-216, 2000.

7 Eisenhauer EA, Therasse P, Therasse J, Bogaerts LH, Schwartz D Sargent R, Ford R, Dancey J, Arbuck S, Gwyther S, Mooney M, Rubinstein L, Shankar L, Dodd L, Kaplan R, Lacombe D and Verweij J: New Response Evaluation Criteria in Solid Tumors: Revised RECIST Guidelines (version 1.1). Eur J Cance 45(2): 228-247, 2009.

8 Choi H, Charnsangavej C, Faria SC, Macapinlac HA, Burgess MA, Patel SR, Chen LL, Podoloff DA and Benjamin RS: Correlation of computed tomography and positron emission tomography in patients with metastatic gastrointestinal stromal tumor treated at a single institution with imatinib mesylate: proposal of new computed tomography response criteria. J Clin Oncol 25(13): 1753-1759, 2007.

9 Dudeck O, Zeile M, Reichardt P and Pink D: Comparison of RECIST and Choi criteria for computed tomographic response evaluation in patients with advanced gastrointestinal stromal tumor treated with sunitinib. Ann Onco 22(8): 1828-1833, 2011.

10 Schramm N, Englhart E and Schlemmer M, Hittinger M, Übleis C, Becker CR, Reiser MF and Berger F: Tumor response and clinical outcome in metastatic gastrointestinal stromal tumors under sunitinib therapy: comparation of RECIST, Choi and volumetric criteria. Eur J Radiol 82(6): 951-958, 2013.

11 Van der Veldt AA, Meijerink MR, van den Eertwegh AJM, Haanen JBA and Boven E: Choi response criteria for early prediction of clinical outcome in patients with metastatic renal cell cancer treated with sunitinib. Br J Cancer 102(5): 803-809, 2010.

12 Ronot M, Bouattour M, Wassermann J, Bruno O, Dreyer C, Larroque B, Castera L, Vilgrain V, Belghiti J, Raymond E and Faivre S: Alternative Response Criteria (Choi, European association for the study of the liver, and modified Response Evaluation Criteria in Solid Tumors [RECIST]) Versus RECIST 1.1 in patients with advanced hepatocellular carcinoma treated with sorafenib. Oncologist 19(4): 394-402, 2014.

13 Weng Z, Ertle J, Zheng S, Lauenstein T, Mueller Bockisch A Gerken G, Yang D and Schlaak JF : Choi criteria are superior in evaluating tumor response in patients treated with transarterial radioembolization for hepatocellular carcinoma. Oncol Lett $6(6)$ : 1707-1712, 2013.

14 Van Cutsem E, Köhne CH, Hitre E Zaluski J, Chien CRC Makhson A D'Haens G, Pintér T, Lim R, Bodoky G, Roh JK, Folprecht G, Ruff P, Stroh C, Tejpar S, Schlichting M, Nippgen $\mathrm{J}$ and Rougier P: Cetuximab and Chemotherapy as Initial Treatment for Metastatic Colorectal Cancer. N Engl J Med 360(14): 1408-1417, 2009.

15 Hurwitz H, Fehrenbacher L, Novotny W, Cartwright T, Hainsworth JHeim W Berlin J, Baron A, Griffing S, Holmgren E, Ferrara N, Fyfe G, Rogers B, Ross R and Kabbinavar F: Bevacizumab plus Irinotecan, Fluorouracil, and Leucovorin for metastatic colorectal cancer. N Engl J Med 350(23): 2335-2342, 2004.

16 Saltz LB, Clarke S, Diaz-Rubio E, Scheithauer W, Figer A, Wong R, Koski S, Lichinitser M, Yang TS, Rivera F, Couture F, Sirzén F and Cassidy J: Bevacizumab in combination with oxaliplatin-based chemotherapy as first-line therapy in metastatic colorectal cancer: a randomized phase III study. J Clin Oncol 26(12): 2013-2019, 2008.

17 Giantonio BJ, Catalano PJ, Meropol NJ, O’Dwyer PJ, Mitchell EP Alberts SR Schwartz MA and Benson AB : Bevacizumab in combination with oxaliplatin, fluorouracil, and leucovorin (FOLFOX4) for previously treated metastatic colorectal cancer: results from the Eastern Cooperative Oncology Group Study E3200. J Clin Oncol 25(12): 1539-1544, 2007.

18 Douillard JY, Siena S, Cassidy J, Tabernero J, Burkes R, Barugel M, Humblet Y, Bodoky G, Cunningham D, Jassem J, Rivera F, Kocákova I, Ruff P, Błasińska-Morawiec M, Smakal M, Canon JL, Rother M, Oliner KS, Wolf M and Gansert : Randomized, phase III trial of Panitumumab with infusional Fluorouracil, Leucovorin, and Oxaliplatin (FOLFOX4) versus FOLFOX4 alone as first-line treatment in patients with previously untreated metastatic colorectal cancer: the PRIME study. J Clin Oncol 28(31): 4697-4705, 2010.

19 Peeters M, Price TJ, Cervantes A, Sobrero AF, Ducreux M, Hotko Y André T, Chan E, Lordick F, Punt CJ, Strickland AH, Wilson G, Ciuleanu TE, Roman L, Van Cutsem E, Tzekova V, Collins S, Oliner KS, Rong A and Gansert J : Randomized phase III study of panitumumab with fluorouracil, leucovorin, and irinotecan (FOLFIRI) compared with FOLFIRI alone as secondline treatment in patients with metastatic colorectal cancer. J Clin Oncol 28(31): 4706-4713, 2010. 
20 Lo Re G, De Luca R, Muscarneri F, Dorangricchia P, Picone D, Vernuccio F, Salerno S, La Tona G, Pinto A, Midiri M, Russo A, Lagalla R and Cicero G: Relationship between anxiety level and radiological investigation. Comparison among different diagnostic imaging exams in a prospective single-center study. Radiologia Medica 121(10): 763-768, 2016.

21 Heinemann V, von Weikersthal LF, Decker T, Kiani A, VehlingKaiser U, Al-Batran SE, Heintges T, Lerchenmüller C, Kahl C, Seipelt G, Kullmann F, Stauch M, Scheithauer W, Hielscher J, Scholz M, Müller S, Link H, Niederle N, Rost A, Höffkes HG, Moehler M, Lindig RU, Modest DP, Rossius L, Kirchner T, Jung A and Stintzing S: FOLFIRI plus cetuximab versus FOLFIRI plus bevacizumab as first-line treatment for patients with metastatic colorectal cancer (FIRE-3): a randomised, open-label, phase 3 trial. Lancet Oncol 15(10): 1065-1075, 2014.
22 Jiang T, Kambadakone A, Kulkarni NM, Zhu AX and Sahani DV: Monitoring response to antiangiogenic treatment and predicting outcomes in advanced hepatocellular carcinoma using image biomarkers, CT perfusion, tumor density, and tumor size (RECIST). Invest Radiol 47(1): 11-17, 2012.

23 Tirkes T, Hollar MA, Tann M, Kohli MD, Akisik F and Sandrasegaran K: Response criteria in oncologic imaging: review of traditional and new criteria. Radiographics 33(5): 1323-1341, 2013. 